\title{
Continuous Generalized Hankel-type integral wavelet transformation
}

\section{R. Lakshmi Gorty}

SVKM's NMIMS University, MPSTME, Department of Basic Science and Humanities, Associate Professor,

\section{ABSTRACT}

Vile-Parle (W), Mumbai -400056, India

Using the theory of Hankel-type convolution, continuous generalized Hankel-type wavelet integral transformation is defined. The generalized Hankel-type integral wavelet transformation is developed. Using the developed theory of generalized Hankel-type convolution, the generalized Hankel-type translation is introduced. Properties of the kernel $D_{\mu, \alpha, \beta, v}(x, y, z)$ are developed in the study. Using the properties of kernel, the generalized Hankel-type wavelet transformation is defined. The existence of the generalized Hankel-type integral wavelet transformation is given by a theorem. The boundedness and inversion formula for the generalized Hankel-type integral wavelet transformation is obtained. A basic wavelet which defines continuous generalized Hankel-type integral wavelet transformation, its admissibility conditions and the wavelet to the function is proved. Examples have been shown to explain the studied continuous generalized Hankel-type integral wavelet transformation.

\section{INDEXING TERMS/KEYWORDS}

Continuous generalized Hankel-type integral wavelet transformation; generalized Hankel-type transformation; Hankel-type Convolution.

\section{SUBJECT CLASSIFICATION}

44A20, 42C40, 46, 33C05

\section{Council for Innovative Research}

Peer Review Research Publishing System

\section{Journal: JOURNAL OF ADVANCES IN MATHEMATICS}

Vol.11, No.3

www.cirjam.com, editorjam@gmail.com 


\section{INTRODUCTION}

Malgonde [1] investigated the following generalized Hankel-type integral transformation

$F_{1}(t)=\left(F_{1, \mu, \alpha, \beta, v} f\right)(t)=v \beta t^{-1-2 \alpha+2 v} \int_{0}^{\infty}(x t)^{\alpha} J_{\mu}\left[\beta(x t)^{v}\right] f(x) d x$,

$J_{\mu}(x)$ being the Bessel function of the first kind of order $\mu \geq-1 / 2$.

We define $L_{p}(0, \infty), 1 \leq p \leq \infty$, as the space of real measurable function $\phi$ on $(0, \infty)$ for which

$\|\phi\|_{\mu, v, p}=\left(\int_{0}^{\infty}\left|x^{\mu v-\alpha} \phi(x)\right|^{p} \frac{d x}{x}\right)^{1 / p}, 1 \leq p<\infty$

$\|\phi\|_{\infty}=\underset{0<x<\infty}{\operatorname{ess} \sup }\left|x^{\mu v-\alpha} \phi(x)\right|<\infty$.

For each $\phi \in L_{1}(0, \infty)$, generalized Hankel-type integral transformation of $\phi$ is defined by

$\hat{\phi}(x)=v \beta t^{-1-2 \alpha+2 v} \int_{0}^{\infty}(x t)^{\alpha} J_{\mu}\left[\beta(x t)^{v}\right] \phi(t) d t, 0<t<\infty$.

From [1] we know that $\hat{\phi}(x)$ is bounded and continuous on $(0, \infty)$ and $\|\hat{\phi}(x)\|_{\infty} \leq\|\phi\|_{1}$.

If $f(x)$ is of bounded variation into a neighborhood of the point $x=x_{0}>0, \mu \geq-1 / 2$ and the integral $\int_{0}^{\infty}|f(x)| x^{\alpha-v / 2}$ exists, then the inversion formula in [2] is given by

$\lim _{R \rightarrow \infty} v \beta y_{0}^{-1-2 \alpha+2 v} \int_{0}^{R}\left(x_{0} y\right)^{\alpha} J_{\mu}\left[\beta\left(x_{0} y\right)^{v}\right] F_{1}(y) d y=\frac{1}{2}\left[f\left(x_{0}+0\right)+f\left(x_{0}-0\right)\right]$.

If $f(x) x^{-\alpha-\mu}$ and $F_{2}(y) y^{\mu-\alpha-1+2 v}$ are in $L_{1}(0, \infty)$, for $F_{1}(t)=\left(F_{1, \mu, \alpha, \beta, v} f(x)\right)(t)=v \beta t^{-1-2 \alpha+2 v} \int_{0}^{\infty}(x t)^{\alpha} J_{\mu}\left[\beta(x t)^{v}\right] f(x) d x$

and

$F_{2}(t)=\left(F_{2, \mu, \alpha, \beta, v} g(x)\right)(t)=v \beta t^{-1-2 \alpha+2 v} \int_{0}^{\infty}(x t)^{\alpha} J_{\mu}\left[\beta(x t)^{v}\right] \mathrm{g}(x) d x$, for $\mu \geq-1 / 2$,

the following mixed Parseval formula holds for $F_{1}$-transformation by [2];

$\int_{0}^{\infty} f(x) g(x) d x=\int_{0}^{\infty} F_{1}(y) F_{2}(y) d y$.

To define the generalized Hankel-type Convolution, we need to introduce generalized Hankel-type translation. Define $D_{\mu, \alpha, \beta, v}(x, y, z)$

$=\int_{0}^{\infty} t^{-\mu v-\alpha} v \beta t^{-1-2 \alpha+2 v}(x t)^{\alpha} J_{\mu}\left[\beta(x t)^{v}\right] v \beta t^{-1-2 \alpha+2 v}(y t)^{\alpha} J_{\mu}\left[\beta(y t)^{v}\right] v \beta t^{-1-2 \alpha+2 v}(z t)^{\alpha} J_{\mu}\left[\beta(z t)^{v}\right] d t$. 
Properties of the kernel $D_{\mu, \alpha, \beta, \nu}(x, y, z)$ :

Following [3] properties are established:

i) For $0<x, y<\infty$ and $0 \leq t<\infty$, we have

$\int_{0}^{\infty} v \beta t^{\mu v+\alpha} z^{-1-2 \alpha+2 v}(z t)^{\alpha} J_{\mu}\left[\beta(z t)^{\nu}\right] D_{\mu, \alpha, \beta, v}(x, y, z) d z=(v \beta)^{2}(x y)^{-1-2 \alpha+2 v}(x t)^{\alpha} J_{\mu}\left[\beta(x t)^{\nu}\right](y t)^{\alpha} J_{\mu}\left[\beta(y t)^{\nu}\right]$

Proof:

$$
\begin{gathered}
D_{\mu, \alpha, \beta, v}(x, y, z)=(v \beta)^{3} \int_{0}^{\infty} t^{-\mu v-\alpha} z^{-1-2 \alpha+2 v}(z t)^{\alpha} J_{\mu}\left[\beta(z t)^{v}\right]\left[x^{-1-2 \alpha+2 v}(x t)^{\alpha} J_{\mu}\left[\beta(x t)^{v}\right] y^{-1-2 \alpha+2 v}(y t)^{\alpha} J_{\mu}\left[\beta(y t)^{v}\right]\right] d z \\
=\int_{0}^{\infty} v \beta t^{-\mu v-\alpha} z^{-1-2 \alpha+2 v}\left[(v \beta)^{2}(x y)^{-1-2 \alpha+2 v}(x t)^{\alpha} J_{\mu}\left[\beta(x t)^{v}\right](y t)^{\alpha} J_{\mu}\left[\beta(y t)^{v}\right]\right](z t)^{\alpha} J_{\mu}\left[\beta(z t)^{v}\right] d z \\
=t^{-\mu v-\alpha} F_{1, \mu, \alpha, \beta, v}\left\{(v \beta)^{2}(x y)^{-1-2 \alpha+2 v}(x t)^{\alpha} J_{\mu}\left[\beta(x t)^{v}\right](y t)^{\alpha} J_{\mu}\left[\beta(y t)^{v}\right]\right\} \\
F_{1, \mu, \alpha, \beta, v}^{-1}\left\{t^{\mu v+\alpha} D_{\mu, \alpha, \beta, v}(x, y, z)\right\}=(v \beta)^{2}(x y)^{-1-2 \alpha+2 v}(x t)^{\alpha} J_{\mu}\left[\beta(x t)^{v}\right](y t)^{\alpha} J_{\mu}\left[\beta(y t)^{v}\right]
\end{gathered}
$$

Applying the inversion formula of generalized Hankel-type integral transformation to (1.2)

$$
\begin{aligned}
& \int_{0}^{\infty} v \beta t^{\mu v+\alpha} z^{-1-2 \alpha+2 v}(z t)^{\alpha} J_{\mu}\left[\beta(z t)^{v}\right] D_{\mu, \alpha, \beta, v}(x, y, z) d z \\
& =(v \beta)^{2}(x y)^{-1-2 \alpha+2 v}(x t)^{\alpha} J_{\mu}\left[\beta(x t)^{v}\right](y t)^{\alpha} J_{\mu}\left[\beta(y t)^{v}\right] .
\end{aligned}
$$

and hence the result. In particular, taking $t=0$, gives

ii) $\int_{0}^{\infty} v \beta t^{\mu v+\alpha} z^{-1-2 \alpha+2 v}(z t)^{\alpha} J_{\mu}\left[\beta(z t)^{v}\right] D_{\mu, \alpha, \beta, v}(x, y, z) d z=1$,

i.e. for which $x, y>0, D_{\mu, \alpha, \beta, \nu}(x, y, z)$ belongs to $L_{0, \alpha, \beta, \nu, \mu}^{1}(0, \infty)$.

iii) $0<x, y, z<\infty, D_{\mu, \alpha, \beta, \nu}(x, y, z) \geq 0$.

iv) $D_{\mu, \alpha, \beta, \nu}(x, y, z)=D_{\mu, \alpha, \beta, \nu}(y, x, z)=D_{\mu, \alpha, \beta, \nu}(z, x, y)=\ldots$

The generalized Hankel-type integral translation $T_{y}$ of $\phi \in L_{p}(0, \infty), 1 \leq p \leq \infty$, is defined by

$T_{y} \phi(x)=\phi(x, y)=\int_{0}^{\infty} \phi(z) D_{\mu, \alpha, \beta, v}(x, y, z) d z, 0<x, y<\infty$.

The map $y \rightarrow T_{y} \phi$ is continuous from $(0, \infty)$ into $(0, \infty)$.

Let $p, q, r \in[1, \infty)$ and $\frac{1}{r}=\frac{1}{p}+\frac{1}{q}-1$. The generalized Hankel-type integral convolution of

$\phi \in L_{p}(0, \infty)$ and $\psi \in L_{p}(0, \infty)$ is defined by $(\phi \# \psi)(x)=\int_{0}^{\infty} \phi(x, y) \psi(y) d y$.

In [4] the integral is convergent for almost all $x, 0<x<\infty$ and $\|\phi \# \psi\|_{r} \leq\|\phi\|_{p}\|\psi\|_{q}$. 
Moreover, $p=\infty$, then $(\phi \# \psi)(x)$ is defined for all $x, 0<x<\infty$ and is continuous.

If $\phi, \psi \in L_{1}(0, \infty)$, then $(\phi \# \psi) \wedge(t)=\hat{\phi}(t) \hat{\psi}(t), 0 \leq t<\infty$.

In this paper, in terms of the aforesaid generalized Hankel-type translation $T_{y}$ and dilation $D_{a}$ defined by

$$
D_{\mu, \alpha, \beta, v, a} \phi(x, y)=a^{-2(\mu v-\alpha)-3 v} \phi(x / a, y / a)
$$

is a continuous generalized Hankel-type integral wavelet transformation is defined. Its continuity and boundedness properties are established. An inversion formula is obtained.

\section{CONTINUOUS GENERALIZED HANKEL-TYPE INTEGRAL WAVELET TRANSFORMATION}

Let $\psi \in L_{p}(0, \infty), 1 \leq p<\infty$ be given. For $b \geq 0$ and $a>0$ define the generalized Hankel-type integral wavelet transformation

$$
\begin{aligned}
\psi_{b, a}(x)=D_{\mu, \alpha, \beta, v, a} T_{y} \psi(x)=D_{\mu, \alpha, \beta, v, a} \psi(b, x) & =a^{-2(\mu v-\alpha)-3 v} \psi(b / a, x / a) \\
& =a^{-2(\mu v-\alpha)-3 v} \int_{0}^{\infty} D_{\mu, \alpha, \beta, v}(b / a, x / a, z) \psi(z) d z
\end{aligned}
$$

the integral being convergent by virtue of [8].

Using the wavelet $\psi_{b, a}$, define the generalized Hankel-type integral wavelet transformation,

$$
\begin{aligned}
H_{1, \alpha, \beta, \nu, \mu}(b, a) & =\left(H_{1, \alpha, \beta, v, \mu, \psi} f\right)(b, a) \\
& =\left\langle f(t), \psi_{b, a}(t)\right\rangle \\
& =\int_{0}^{\infty} f(t) \overline{\psi_{b, a}(t)} d t \\
& =a^{-2(\mu v-\alpha)-3 v} \int_{0}^{\infty} \int_{0}^{\infty} f(t) \overline{\psi(z)} D_{\mu, \alpha, \beta, v}(b / a, t / a, z) d z d t
\end{aligned}
$$

provided the integral is convergent.

The continuity of the generalized Hankel-type integral wavelet follows from the boundedness property of the generalized Hankel-type translation [5].

Lemma 1: Let $\psi \in L_{p}(0, \infty), 1 \leq p<\infty$. Then for $y \geq 0$, the map $y \rightarrow T_{y} f$ is continuous from $L_{p}(0, \infty)$ into $L_{p}(0, \infty)$. The function $\psi_{b, a}$ is defined almost everywhere on $[0, \infty)$, and $\left\|\psi_{b, a}(x)\right\|_{p} \leq a^{(2(\mu v-\alpha)+3 v)(1 / p-1)}\|\psi\|_{p}$.

The existence of the generalized Hankel-type transformation is given by the following theorem.

Theorem 2. Let $f \in L_{p}(0, \infty)$ and $\psi \in L_{p}(0, \infty)$ with $1 \leq p, q<\infty$ and $\frac{1}{p}+\frac{1}{q}=1 ; H_{1, \alpha, \beta, \nu, \mu}(b, a)=\left(H_{1, \alpha, \beta, \nu, \mu, \psi} f\right)(b, a)$ be the continuous wavelet transform. Then

1) $\left(H_{1, \alpha, \beta, \nu, \mu} f\right)(b, a)$ is continuous on $(0, \infty) \times(0, \infty)$,

2) $\left\|\left(\left(H_{1, \alpha, \beta, v, \mu, \psi} f\right)(b, a) f\right)(b, a)\right\|_{r} \leq a^{2(\mu v-\alpha)+3 v}\|f\|_{p}\|\psi\|_{q}, \frac{1}{r}=\frac{1}{p}+\frac{1}{q}-1,1 \leq p, q, r<\infty$,

3) $\left\|\left(\left(H_{1, \alpha, \beta, v, \mu, \psi} f\right)(b, a) f\right)(b, a)\right\|_{\infty} \leq a^{(2(\mu v-\alpha)+3 v)\left(\frac{1}{q}-1\right)}\|f\|_{p}\|\psi\|_{q}, \frac{1}{p}+\frac{1}{q}=1$. 


\section{Proof.}

1) Let $\left(b_{0}, a_{0}\right)$ be an arbitrary but fixed point in $(0, \infty) \times(0, \infty)$. Then by Hölder's inequality,

$$
\begin{aligned}
\mid\left(H_{1, \alpha, \beta, \nu, \mu} f\right)(b, a)- & \left(H_{1, \alpha, \beta, v, \mu} f\right)\left(b_{0}, a_{0}\right) \mid \\
\leq & a^{-2(\mu v-\alpha)-3 v} \int_{0}^{\infty} \int_{0}^{\infty}\left|f(t) \psi(z)\left[D_{\mu, \alpha, \beta, \nu}(b / a, t / a, z)-D_{\mu, \alpha, \beta, v}\left(b_{0} / a_{0}, t / a_{0}, z\right)\right]\right| d t d z \\
\leq & a^{-2(\mu v-\alpha)-3 v}\left[\int_{0}^{\infty} \int_{0}^{\infty}|f(t)|^{p}\left|D_{\mu, \alpha, \beta, \nu}(b / a, t / a, z)-D_{\mu, \alpha, \beta, v}\left(b_{0} / a_{0}, t / a_{0}, z\right)\right| d t d z\right]^{1 / p} \\
& \times\left[\int_{0}^{\infty} \int_{0}^{\infty}|\psi(z)|^{q}\left|D_{\mu, \alpha, \beta, v}(b / a, t / a, z)-D_{\mu, \alpha, \beta, v}\left(b_{0} / a_{0}, t / a_{0}, z\right)\right| d t d z\right]^{1 / q}
\end{aligned}
$$

Since by (9) $\int_{0}^{\infty}\left|D_{\mu, \alpha, \beta, \nu}(b / a, t / a, z)-D_{\mu, \alpha, \beta, \nu}\left(b_{0} / a_{0}, t / a_{0}, z\right)\right| d t \leq 2$, by dominated convergence theorem and continuity of $D_{\mu, \alpha, \beta, v}(b / a, t / a, z)$ in the variables $b$ and $a$, we have $\lim _{\substack{b \rightarrow b_{0} \\ a \rightarrow a_{0}}}\left|\left(H_{1, \alpha, \beta, v, \mu} f\right)(b, a)-\left(H_{1, \alpha, \beta, v, \mu} f\right)\left(b_{0}, a_{0}\right)\right|=0$. This proves that $H(b, a)$ is continuous on $(0, \infty) \times(0, \infty)$

2) $\quad\left\|\left(\left(H_{1, \alpha, \beta, v, \mu, \psi} f\right)(b, a) f\right)(b, a)\right\|_{r} \leq a^{2(\mu v-\alpha)+3 v}\|f\|_{p}\|\psi\|_{q}, \frac{1}{r}=\frac{1}{p}+\frac{1}{q}-1,1 \leq p, q, r<\infty$.

3) It can be proved using Hölder's inequality.

\section{AN INVERSION FORMULA}

In this section, we show that the function $f$ can be recovered from its wavelet transform when the wavelet $\psi$ satisfies admissibility condition.

Theorem 3. Let $\psi \in L^{2}\left(\mathrm{R}_{+}\right)$be a basic wavelet which defines generalized Hankel-type wavelet integral transformation. Then, for $A_{\psi}=\int_{0}^{\infty} w^{-2(\mu v-\alpha)-3 v}|\hat{\psi}(w)|^{2} d w>0$, we have

$$
\begin{array}{r}
\int_{0}^{\infty} \int_{0}^{\infty}\left(\left(H_{1, \alpha, \beta, v, \mu, \psi} f\right)(b, a) f\right)(b, a) \overline{\left(\left(H_{1, \alpha, \beta, \nu, \mu, \psi} f\right)(b, a) g\right)(b, a)} a^{-2(\mu v-\alpha)-3 v} d a d b \\
=A_{\psi}\langle f, g\rangle \text { for all } f, g \in L^{2}\left(\mathrm{R}_{+}\right) .
\end{array}
$$

Proof. The representation for $\left(H_{1, \alpha, \beta, \nu, \mu, \psi} f\right)(b, a)$, can be expressed as

$$
\begin{aligned}
& \left(H_{1, \alpha, \beta, \nu, \mu, \psi} f\right)(b, a) \\
& =\int_{0}^{\infty} \int_{0}^{\infty} f(t) \overline{\psi(z)} D_{\mu, \alpha, \beta, \nu}(b / a, t / a, z) d z d t
\end{aligned}
$$




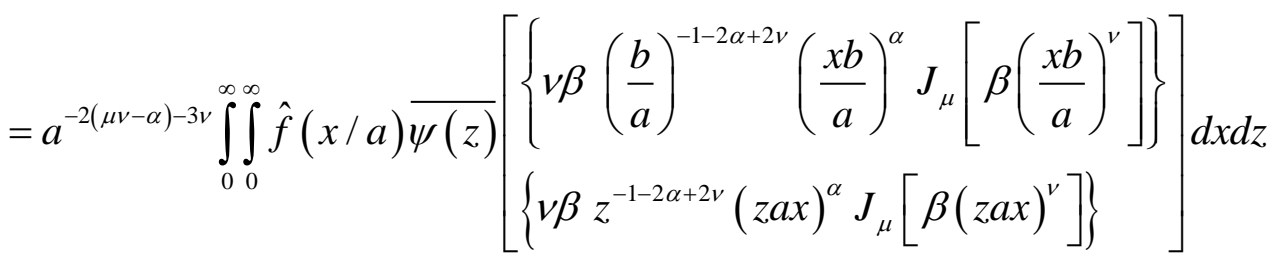

$$
\begin{aligned}
& =a^{-2(\mu v-\alpha)-3 v} \int_{0}^{\infty} \hat{f}(x / a) \overline{\hat{\psi}(x)}\left\{v \beta\left(\frac{b}{a}\right)^{-1-2 \alpha+2 v}\left(\frac{x b}{a}\right)^{\alpha} J_{\mu}\left[\beta\left(\frac{x b}{a}\right)^{v}\right]\right\} d x \\
& =\int_{0}^{\infty} \hat{f}(u) \overline{\hat{\psi}(a u)}\left\{v \beta b^{-1-2 \alpha+2 v}(b u)^{\alpha} J_{\mu}\left[\beta(b u)^{v}\right]\right\} d u \\
& =(\hat{f}(u) \overline{\hat{\psi}(a u)}) \wedge(b) \text {. }
\end{aligned}
$$

Parseval identity yields

$$
\begin{aligned}
& \int_{0}^{\infty}\left(H_{1, \alpha, \beta, \nu, \mu, \psi} f\right)(b, a) \overline{\left(H_{1, \alpha, \beta, \nu, \mu, \psi} f\right)(b, a)} d b \\
& =\int_{0}^{\infty}(\hat{f}(u) \overline{\hat{\psi}(a u)}) \wedge(b) \overline{(\hat{g}(u) \overline{\hat{\psi}(a u)})} \wedge(b) d b \\
& =\int_{0}^{\infty}(\hat{f}(u) \overline{\hat{\psi}(a u)}) \overline{(\hat{g}(u) \overline{\hat{\psi}(a u)})} d u .
\end{aligned}
$$

Now multiplying by $a^{-2(\mu v-\alpha)-3 v} d a$ and integrating, we get

$$
\begin{aligned}
\int_{0}^{\infty} \int_{0}^{\infty}\left(H_{1, \alpha, \beta, v, \mu, \psi} f\right)(b, a) \overline{\left(H_{1, \alpha, \beta, v, \mu, \psi} f\right)(b, a)} d b a^{-2(\mu v-\alpha)-3 v} d a \\
\quad=\int_{0}^{\infty} \int_{0}^{\infty}(\hat{f}(u) \overline{\hat{\psi}(a u)}) \overline{(\hat{g}(u) \overline{\hat{\psi}(a u)})} d u \cdot a^{-2(\mu v-\alpha)-3 v} d a \\
\quad=\int_{0}^{\infty} \hat{f}(u) \overline{\hat{g}(u)} d u \int_{0}^{\infty} \hat{\psi}(a u) \overline{\hat{\psi}(a u)} \cdot a^{-2(\mu v-\alpha)-3 v} d a \\
=\int_{0}^{\infty} \hat{f}(u) \overline{\hat{g}(u)} d u \int_{0}^{\infty}|\hat{\psi}(a u)|^{2} \cdot a^{-2(\mu v-\alpha)-3 v} d a \\
=\int_{0}^{\infty} \hat{f}(u) \overline{\hat{g}(u)} d u \int_{0}^{\infty}|\hat{\psi}(w)|^{2} \cdot w^{-2(\mu v-\alpha)-3 v} d w \\
=A_{\psi}\langle f, g\rangle .
\end{aligned}
$$

Notice that admissibility condition requires that $\hat{\psi}(0)=0$. If $\hat{\psi}$ is continuous, it follows that $\int_{0}^{\infty} \psi(x) d x=0$. This justifies the wavelet to the function. 
Now consider

$\psi\left(\frac{b}{a}, \frac{t}{a}\right)=\psi(x, y)$ by putting $\frac{b}{a}=x$ and $\frac{t}{a}=y$. Then $\psi(x, y)=\int_{0}^{\infty} \psi(z) D_{\mu, \alpha, \beta, \nu}(b / a, t / a, z) d z$.

Since

$$
D_{\mu, \alpha, \beta, v}(x, y, z)=\int_{0}^{\infty} \xi^{-\mu v-\alpha}\left[\begin{array}{l}
v \beta x^{-1-2 \alpha+2 v}(x \xi)^{\alpha} J_{\mu}\left[\beta(x \xi)^{v}\right] \\
v \beta y^{-1-2 \alpha+2 v}(y \xi)^{\alpha} J_{\mu}\left[\beta(y \xi)^{v}\right] v \beta z^{-1-2 \alpha+2 v}(z \xi)^{\alpha} J_{\mu}\left[\beta(z \xi)^{v}\right] d \xi
\end{array}\right] .
$$

Substituting the expression becomes

$$
\begin{aligned}
\psi(x, y) & =\int_{0}^{\infty} \psi(z)\left[\int_{0}^{\infty} \xi^{-\mu v-\alpha}\left\{\begin{array}{l}
v \beta x^{-1-2 \alpha+2 v}(x \xi)^{\alpha} J_{\mu}\left[\beta(x \xi)^{v}\right] \\
v \beta y^{-1-2 \alpha+2 v}(y \xi)^{\alpha} J_{\mu}\left[\beta(y \xi)^{v}\right] \\
v \beta z^{-1-2 \alpha+2 v}(z \xi)^{\alpha} J_{\mu}\left[\beta(z \xi)^{v}\right] d \xi
\end{array}\right\} d z\right. \\
& =\int_{0}^{\infty}\left(\int_{0}^{\infty} \psi(z) v \beta z^{-1-2 \alpha+2 v}(z \xi)^{\alpha} J_{\mu}\left[\beta(z \xi)^{v}\right] d z\right) \xi^{-\mu v-\alpha}\left\{\begin{array}{l}
v \beta x^{-1-2 \alpha+2 v}(x \xi)^{\alpha} J_{\mu}\left[\beta(x \xi)^{v}\right] \\
v \beta y^{-1-2 \alpha+2 v}(y \xi)^{\alpha} J_{\mu}\left[\beta(y \xi)^{v}\right]
\end{array}\right\} d \xi \\
& =\int_{0}^{\infty} \xi^{-\mu v-\alpha}\left\{v \beta x^{-1-2 \alpha+2 v}(x \xi)^{\alpha} J_{\mu}\left[\beta(x \xi)^{v}\right] v \beta y^{-1-2 \alpha+2 v}(y \xi)^{\alpha} J_{\mu}\left[\beta(y \xi)^{v}\right]\right\}\left(F_{1, \mu, \alpha, \beta, \nu} \psi\right)(\xi) d \xi .
\end{aligned}
$$

Substitute $\frac{b}{a}=x$ and $\frac{t}{a}=y$.

$\psi\left(\frac{b}{a}, \frac{t}{a}\right)=\int_{0}^{\infty} \xi^{-\mu v-\alpha}\left\{v \beta\left(\frac{b}{a}\right)^{-1-2 \alpha+2 v}\left(\frac{b \xi}{a}\right)^{\alpha} J_{\mu}\left[\beta\left(\frac{b \xi}{a}\right)^{v}\right] v \beta\left(\frac{t}{a}\right)^{-1-2 \alpha+2 v}\left(\frac{t \xi}{a}\right)^{\alpha} J_{\mu}\left[\beta\left(\frac{t \xi}{a}\right)^{v}\right]\right\}\left(F_{1, \mu, \alpha, \beta, v} \psi\right)(\xi) d \xi$.

$\left(H_{1, \alpha, \beta, \nu, \mu, \psi} f\right)(b, a)$

$=a^{-2(\mu \nu-\alpha)-3 v} \int_{0}^{\infty} \psi\left(\frac{b}{a}, \frac{t}{a}\right) f(t) d t$

$=a^{-2(\mu v-\alpha)-3 v} \int_{0}^{\infty}\left(\int_{0}^{\infty} \xi^{-\mu v-\alpha}\left\{\begin{array}{l}v \beta\left(\frac{b}{a}\right)^{-1-2 \alpha+2 v}\left(\frac{b \xi}{a}\right)^{\alpha} J_{\mu}\left[\beta\left(\frac{b \xi}{a}\right)^{v}\right] \\ v \beta\left(\frac{t}{a}\right)^{-1-2 \alpha+2 v}\left(\frac{t \xi}{a}\right)^{\alpha} J_{\mu}\left[\beta\left(\frac{t \xi}{a}\right)^{v}\right]\end{array}\right\}\left(F_{1, \mu, \alpha, \beta, v} \psi\right)(\xi) d \xi\right) \times f(t) d t$.

$=a^{-2(\mu v-\alpha)-3 v} \int_{0}^{\infty} v \beta\left(\frac{b}{a}\right)^{-1-2 \alpha+2 v}\left(\frac{b \xi}{a}\right)^{\alpha} J_{\mu}\left[\beta\left(\frac{b \xi}{a}\right)^{v}\right]\left(\int_{0}^{\infty} v \beta\left(\frac{t}{a}\right)^{-1-2 \alpha+2 v}\left(\frac{t \xi}{a}\right)^{\alpha} J_{\mu}\left[\beta\left(\frac{t \xi}{a}\right)^{v}\right] f(t) d t\right)$

$\times \xi^{-\mu \nu-\alpha}\left(F_{1, \mu, \alpha, \beta, \nu} \psi\right)(\xi) d \xi$

$=a^{-2(\mu v-\alpha)-3 v} \int_{0}^{\infty} v \beta\left(\frac{b}{a}\right)^{-1-2 \alpha+2 v}\left(\frac{b \xi}{a}\right)^{\alpha} J_{\mu}\left[\beta\left(\frac{b \xi}{a}\right)^{v}\right]\left(F_{1, \mu, \alpha, \beta, v} f\right)\left(\frac{\xi}{a}\right) \times \xi^{-\mu v-\alpha}\left(F_{1, \mu, \alpha, \beta, \nu} \psi\right)(\xi) d \xi$.

By substituting $\frac{\xi}{a}=x ; d \xi=a d x$, the continuous generalized Hankel-type integral wavelet transform can be written as 


$$
\begin{aligned}
& \left(H_{1, \alpha, \beta, \nu, \mu, \psi} f\right)(b, a)=a^{-2(\mu v-\alpha)-3 v} \int_{0}^{\infty} v \beta\left(\frac{b}{a}\right)^{-1-2 \alpha+2 v}\left(\frac{b \xi}{a}\right)^{\alpha} J_{\mu}\left[\beta\left(\frac{b \xi}{a}\right)^{\nu}\right]\left(F_{1, \mu, \alpha, \beta, v} f\right)\left(\frac{\xi}{a}\right) \times \xi^{-\mu v-\alpha}\left(F_{1, \mu, \alpha, \beta, V} \psi\right)(\xi) d \xi . \\
& =a^{-2(\mu \nu-\alpha)-3 v} \int_{0}^{\infty} v \beta\left(\frac{b}{a}\right)^{-1-2 \alpha+2 v}(b x)^{\alpha} J_{\mu}\left[\beta(b x)^{\nu}\right]\left(F_{1, \mu, \alpha, \beta, V} f\right)(x) \times(a x)^{-\mu v-\alpha}\left(F_{1, \mu, \alpha, \beta, \nu} \psi\right)(a x) a d x \\
& =a^{-2(\mu v-\alpha)-3 v} a^{-\mu v-\alpha+(1-2 v+2 \alpha)-1 / 2+1} \int_{0}^{\infty} v \beta b^{-1-2 \alpha+2 v}(b x)^{\alpha} J_{\mu}\left[\beta(b x)^{\nu}\right]\left(F_{1, \mu, \alpha, \beta, v} f\right)(x) x^{-\mu v-\alpha}\left(F_{1, \mu, \alpha, \beta,} \psi\right)(a x) d x \\
& =a^{-2(\mu v-\alpha)-3 v} a^{-\mu \nu+\alpha-2 v+\frac{3}{2}} \int_{0}^{\infty} v \beta b^{-1-2 \alpha+2 v}(b x)^{\alpha} J_{\mu}\left[\beta(b x)^{\nu}\right]\left(F_{1, \mu, \alpha, \beta, V} f\right)(x) x^{-\mu v-\alpha}\left(F_{1, \mu, \alpha, \beta, V} \psi\right)(a x) d x . \\
& =a^{-3 \mu v+3 \alpha-5 v+\frac{3}{2}} \int_{0}^{\infty} v \beta b^{-1-2 \alpha+2 v}(b x)^{\alpha} J_{\mu}\left[\beta(b x)^{\nu}\right]\left(F_{1, \mu, \alpha, \beta, V} f\right)(x) x^{-\mu v-\alpha}\left(F_{1, \mu, \alpha, \beta,} \psi\right)(a x) d x . \\
& D_{\mu, \alpha, \beta, \nu}(x, y, z)=\int_{0}^{\infty} \xi^{-\mu \nu-\alpha}\left[\begin{array}{l}
v \beta x^{-1-2 \alpha+2 v}(x \xi)^{\alpha} J_{\mu}\left[\beta(x \xi)^{\nu}\right] \\
v \beta y^{-1-2 \alpha+2 v}(y \xi)^{\alpha} J_{\mu}\left[\beta(y \xi)^{\nu}\right] \\
v \beta z^{-1-2 \alpha+2 v}(z \xi)^{\alpha} J_{\mu}\left[\beta(z \xi)^{\nu}\right] d \xi
\end{array}\right] \\
& x=\frac{b}{a} ; y=\frac{t}{a} \\
& \xi=a x ; d \xi=a d x . \\
& D_{\mu, \alpha, \beta, \nu}(b / a, t / a, z)=\int_{0}^{\infty}(a x)^{-\mu \nu-\alpha} \nu \beta\left(\frac{t}{a}\right)^{-1-2 \alpha+2 v}(t x)^{\alpha} J_{\mu}\left[\beta(t x)^{\nu}\right] \\
& v \beta z^{-1-2 \alpha+2 v}(z a x)^{\alpha} J_{\mu}\left[\beta(z a x)^{v}\right] a d x \\
& v \beta\left(\frac{b}{a}\right)^{-1-2 \alpha+2 v}(b x)^{\alpha} J_{\mu}\left[\beta(b x)^{v}\right] \\
& D_{\mu, \alpha, \beta, \nu}(b / a, t / a, z)=\int_{0}^{\infty}(a x)^{-\mu \nu-\alpha} v \beta\left(\frac{t}{a}\right)^{-1-2 \alpha+2 v}(t x)^{\alpha} J_{\mu}\left[\beta(t x)^{\nu}\right] \\
& \left.v \beta z^{-1-2 \alpha+2 v}(z a x)^{\alpha} J_{\mu}\left[\beta(z a x)^{\nu}\right] a d x\right]
\end{aligned}
$$




\section{CONCLUSION}

The applications of generalized Hankel-type integral wavelet transformation can be applied in signal processing, computer vision, seismology, turbulence, computer graphics, image processing, digital communication, approximation theory, numerical analysis and statistics.

\section{ACKNOWLEDGMENTS}

I am thankful for the support to this work by National Board of Higher Mathematics Ref No. 2/48(11) 2013/ NBHM/ (R.P.) / R\& D II/979.

\section{REFERENCES}

[1] S. P. Malgonde, 2007. Real Inversion Formula for the distributional generalized Hankel type integral transformation, Rev. Acad. Canaria Cienc, XVIII, Spain.

[2] S. P. Malgonde,2000. On the generalized Hankel-type integral transformation of generalized functions, Indian Journal of pure appl. Math., 31(2):Feb.2000, 197-206.

[3] Watson G. N., 1958. A treatise on the theory of Bessel functions, Cambridge University Press, London.

[4] S. P. Malgonde G. S. Gaikawad; 2001. On a generalized Hankel type convolution of generalized functions, Proc. Indian Acad. Sci. (Math.sci.), Vol.111, No.4, Nov.2001, pp.471-487.

[5] A. H. Zemanian, 1968, Generalized Integral Transformations; Inter Science Publishers, New York.

[6] D. T. Haimo, 1965, "Integral Equations Associated with Hankel convolutions", Trans. Amer. Math. Soc. 116, 33-375.

[7] R. S. Pathak, 2009, The Wavelet Transform, Atlantis Studies in Mathematics for Engineering and Science: Atlantis Press, Vol. 4.

[8] V. R. Lakshmi Gorty, 2013. Continuous generalized Hankel-type wavelet transformation, Universal Journal of Applied Mathematics, Vol. 1 No. 4, Dec 2013, pp. $220-229$.

\section{Author' biography with Photo}

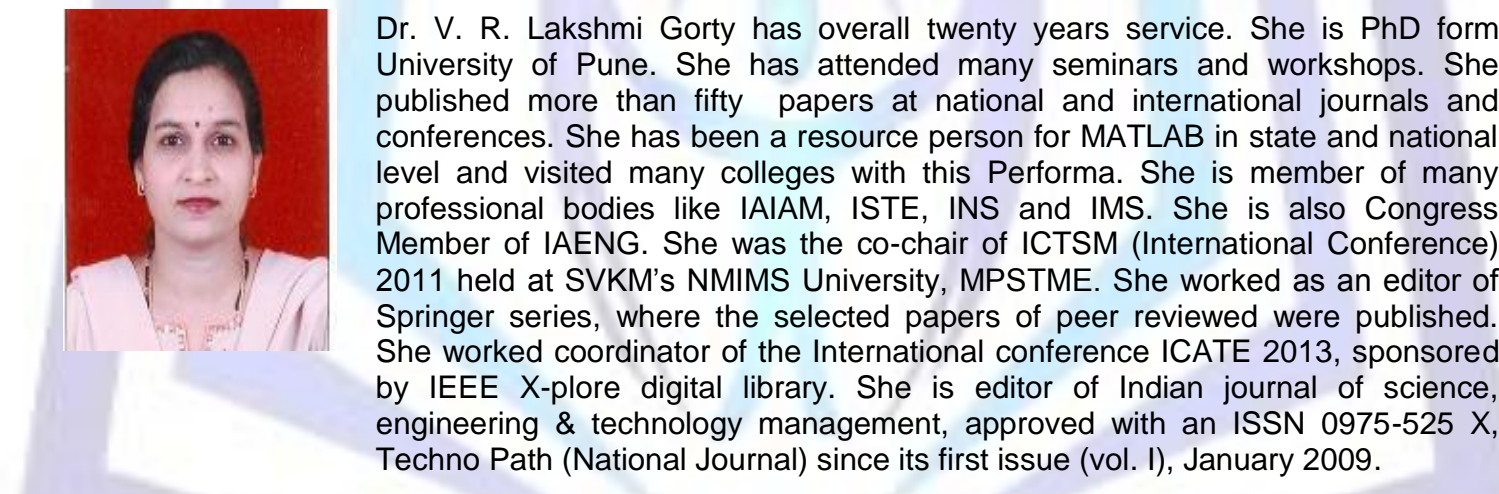

